\title{
Special Chapter in the recent advancement in Brazil in the field of Thermal Analysis and Calorimetry
}

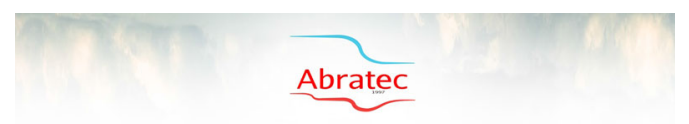

9 a 12 de Novembro de 2014

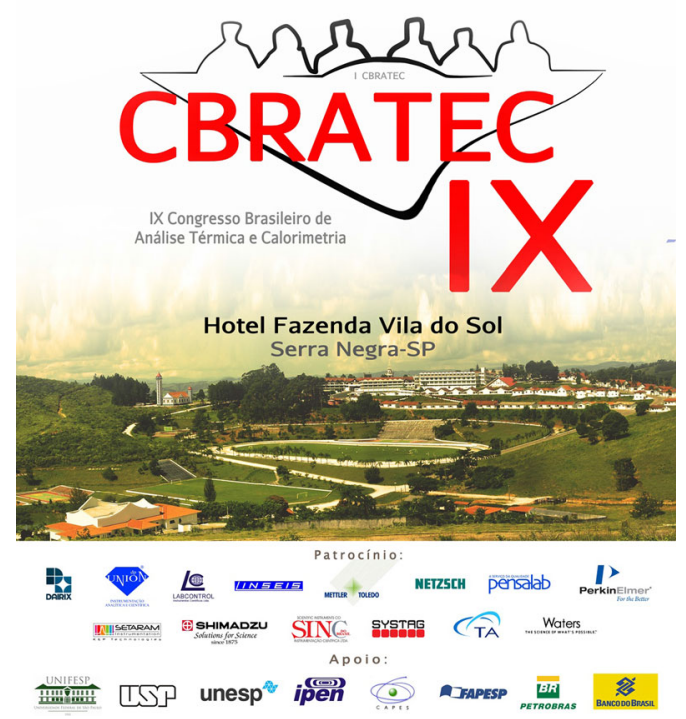

Guest Editor

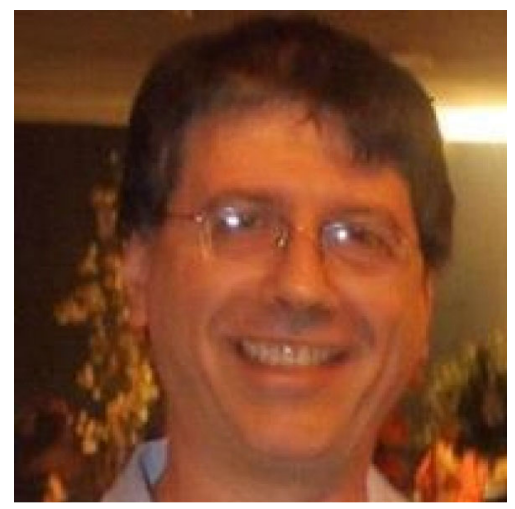

Prof. Dr. Fernando L. Fertonani

Universidade Estadual Paulista Júlio de Mesquita Filho-IBILCE-UNESP fertonan@ibilce.unesp.br

This compilation is based on the peer-reviewed and selected papers presented at IXCBRATEC 


\section{Preface}

\section{Fernando L. Fertonani}

It is my pleasure to present this Special Chapter of Journal of Thermal Analysis and Calorimetry (JTAC) from the IX Brazilian Congress on Thermal Analysis and Calorimetry (CBRATEC-9). The compilation of thirteen peer-reviewed papers is published in this Special Chapter.

The CBRATEC-9 Congress was held in Serra Negra, São Paulo State, November 09-12, 2014. The conference venue was: Hotel Fazenda Vale do Sol, a wonderful place, where all of the participants have been accommodated. This edition of CBRATEC-9 included 210 presentations and more than 300 participants. A wide range of topics in thermal analysis and its applications were covered in 09 sessions, including: A-Food, Pharmaceutical and Life Sciences-Dr. Eder T. G. Cavalheiro/Dr. Marcelo Kobelnik; B-Earth Sciences and Environment, Dr. Sonia de Almeida; C-Kinetics and Catalysis, Dr. Jivaldo R. Matos; D-Combustíveis Fósseis, Biocombustíveis e Explosivos, Dr. Cheila G. Mothé/Dr. Leandro C. Morais; E-Nanomaterials and biomaterials, Dr. Aldo E. Job/Dr. Ana M. Rocco; F-Inorganic chemistry, metallurgy and ceramics, Dr. Hernane S. Barud; G-Organic chemicals, petrochemicals and polymers, asphalt, Dr. Luci D. B. Machado; $\mathrm{H}$-Thermodynamics and calorimetry, thermochemistry, calorimetry, Dr. Lucildes P. Mercuri; I-Others, Dr. José R. Turquetti. In the Opening Ceremony honored the Prof. Dr. Fernando Luis Fertonani (DQCA-IBILCE-UNESP) due to the excellent work as the president.
The Conference started with plenary lecture read by Prof. Dr. Maria Filomena Camões, University of Lisbon (Former President of Analytical Chemistry Division of IUPAC and EURACHEM). Furthermore other nine plenary lectures were accomplished during the Event by the scientists specially invited: Dr. Alfréd Kállay Menyhárd, JTAC Editor-in-Chief, Hungary; Dr. Jo Dweck, Brazil; Dr. Vera Regina Leopoldo Constantino, Brazil; Dr. Ralf Zimmermann, Germany; Dr. Peter Simon, Slovak Republic; Dr. Luis A. Pérez-Maqueda, Spain; Dr. Dirk Walter, Germany; Dr. Win de Klerk, President of ICTAC, United States; Dr. Agnieszka Tercjak, Spain; Dr. Jorma Hölsä, Finland.

I would like to acknowledge Prof. Dr. Alfréd KállayMenyhárd (Editor-in-Chief), Dr. Sophie Korda, and the staff in JTAC for providing support to complete this Chapter. I thank reviewers for their professional evaluation and dedication. Finally, I thank all of the authors for their effort and contribution.

It was decided that the CBRATEC-10 and IV Pan-American Congress on Thermal Analysis and Calorimetry (IV CPANATEC) will be held in the city of São Paulo, Capital of Sao Paulo State, 17-21 April, 2016; venue: Braston Hotel.

Last but not least, let me take this opportunity to express my sincere thanks for all who have ever helped us during the work of organization, for the Sponsors whose financial help was essential and for all participants and accompanying persons who attended the CBRATEC-9.
Fernando L. Fertonani

fertonan@ibilce.unesp.br

1 Universidade Estadual Paulista Júlio de Mesquita Filho-IBILCE-UNESP, São José do Rio Preto, Brazil 


\section{Organize Committee}

President: Fernando L. Fertonani

Vice President: Cheila G. Mothé

Treasurer: Jivaldo do R. Matos

Secretary: Gledison R. de Souza

Assistant of Secretary: Carina M. de Souza

Presidential assistant: Airton J. Damaceno

ICTAC Abratec's representative: Luci D. B. Machado

\begin{tabular}{ll}
\hline Adriano A. Araújo & José R. Turquetti \\
Airton J. Damaceno & Lilian D. de M. Torquato \\
Antonio G. de Souza & Lucas Alves Martins \\
Bruno T. Franzin & Lucildes P. Mercuri \\
Cecilia C. M. dos Santos & Marcelo A. G. Bardi \\
Cibele R. R. de C. Lima & Marcelo Kobelnik \\
Clovis A. Ribeiro & Marcos A. Segatto Silva \\
Cristiane Quinelato & Marisa S. Crespi \\
Douglas L. Casemiro & Michelle Gonçalves \\
Eduardo T. de Almeida & Natália R. Segismundo \\
Elder M. Filho & Rafael dos S. Macedo \\
Filipe C. Guizellini & Simone G. de Ávila \\
Gustavo G. Fontanari & Sônia de Almeida \\
Iêda Ap. Pastre & Vagner Roberto Magri \\
\hline
\end{tabular}

Organize Committee of Companies Sponsors

dpUNION/Hitachi-Abel Ribeiro

Mettler Toledo-Pierre Delmorio

NETZSCH do Brasil—João Y. Mesquita

PerkinElmer do Brasil-Thiago Fatobene

Waters-TA Instruments-Frederico Mendes Jr.

Petrobras-Alessandra R. Cassella

Sinc do Brasil-Marcelo Borghi

SR Grupo-Carlos Braga
Scientific Committee

Adriano A. de Araújo

Alan Riga

Aldo Eloizo Job

Altivo Pitaluga Jr.

Antonio E. Mauro

Antonio Gouveia Souza

Arnaud V. dos Santos

Cecilia C. M. dos Santos

Cheila G. Mothé

Clovis A. Ribeiro

Csaba Novak

Eder T. Cavalheiro

Eduardo T. de Almeida

Fernando L. Fertonani

Gabriel L. B. de Araujo

Gisele C. Becher Ribas

Gustavo G. Fontanari

Hélio Salvio Neto

Hernane da Silva Barud

Iêda A. Pastre

Jean Rouquerol
Jivaldo do R. Matos

José Marques Luiz

José P. Batistuti

José R. Delbem

José R. Turquetti

Judit Simon

Luci D. B. Machado

Lucildes P. Mercuri

Marcelo A. G. Bardi

Marcelo Kobelnik

Maria Luisa A. Gonçalves

Marisa S. Crespi

Najla M. Kassab

Paula Manoel Crakovic

Pedro O. D. Lozano

Peter Simon

Rosa Nomen

Rui Oliveira Macedo

Selma Cunha Mello

Sonia de Almeida

Vera R. L. Constantino 


\section{Acknowledgements}

The Guest Editor is very thankful to the following scientists whose official reviews have significantly contributed to the quality of the papers in this Special Chapter.

Allahbakhsh, A., Iran

Amin, M. El-S., Egypt

Bannach, G., Brazil

Barreto Gomes, A. P., Brazil

Bikiaris, D., Greece

Cai, J., China

Caillat, S., France

Cardoso Morais, L., Brazil

Cavallaro, G., Italy

Crespi, M. S., Brazil

de Guzzi Plepis, A. M., Brazil

Doraiswamy, D., USA

Ebrahimi, M., Iran

Fokaides, P. A., Cyprus

Garcia Trevisan, M., Brazil

Goindi, S., India

Heris, S. Z., Iran

Jiang, X., China

Kozłowska, M., Poland

Lacerda, L., Brazil

Liu, X., China

Majid, K., India

Malucelli, G., Italy

Marcinkowska, A., Poland

Mitovski, A., Serbia

Murta Valle, M. L., Brazil
Nowicki, A. L., Poland

Olar, R., Romania

Paszkiewicz, S., Poland

Patel, T. M., India

Pérez-Maqueda, L., Spain

Perlovich, G., Russian Federation

Pineda, E. A. G., Brazil

Procópio, J. V. V., Brazil

Reis Albuquerque, A., Brazil

Ribeiro, C. A., Brazil

Sanchez-Jimenez, P., Spain

Silva Leonardo, R., Brazil

Souza, A. L., Brazil

Spirandelli Crespi, M., Brazil

Szabó-Révész, P., Hungary

Taraba, B., Czech Republic

Tonsuaadu, K., Estonia

Trzos, M., Poland

Vinu, R., India

Vlaev, L., Bulgaria

Wang, S., China

Wang, S. B., China

Wesolowski, M., Poland

Wong, T. W., Malaysia

Zhai, Y., China

Zhu, F., China 